\title{
OP-Tische, Patientenvorbereitung und Lagerungstechniken
}

\author{
Dankward Höntzsch
}

\section{Zusammenfassung}

OP-Tische, Patientenvorbereitung und Lagerung sind eine typische Schnittstelle im OP zwischen OP-Pflege und Ärzteteam (vor allem Chirurgen, aber auch Anästhesisten). Deshalb ist hier Teamarbeit gefragt. Gerade bei der heute notwendigen Leistungsverdichtung ist dies sehr wichtig. Jeder muss über die technische Ausrüstung der OP-Tische und die Vorbereitungsund Lagerungsmöglichkeiten und
Notwendigkeiten des Patienten Bescheid wissen. In Planspielen und genau vorgegebenen Richtlinien muss dies organisatorisch vorbereitet sein, damit im Tagesablauf alles zusammenspielen kann. Die Wechselzeiten können durch ein einvernehmliches, harmonisches Zusammenspiel aller Kräfte reduziert werden. Hierzu müssen die Bereiche OP-Tische, Patientenvorbereitung und Lagerungstechniken von OP-Pflegepersonal und Ärzten beherrscht werden.

\section{OP-Tische}

Es stehen verschiedene OP-Tischsysteme zur Verfügung. Heute sind OP-Tisch-Säule und Auflagefläche meist getrennt. Aber auch die Vorgängergeneration ist noch in Betrieb. Hier handelt es sich um fahrbare OP-Tische mit Fahrwerk, Säule und Auflagefläche in einem nicht trennbaren System (Abb.1). Diese können im OP auf zweierlei Weise verwendet werden: a) Der OP-Tisch wird direkt an die OPSchleuse und Umlagerung gefahren und von dort in den OP-Saal. Diese OP-Tische haben ein schweres Fahrgestell mit kleinen Laufrädern. Das macht das Fahren schwer und ist für den Boden, die Fugen und die Rillen im OPFlur eine große Belastung. Es kommt zum vorzeitigen Verschleiß, besonders im Bereich von Fugen und Dehnfugen. Die BG Unfallklinik Tübingen kann wie auch andere OP-Abteilungen ein Lied davon singen.
OP-JOURNAL 2005; 21: 52-57

(c) Georg Thieme Verlag KG Stuttgart · New York
Oder:

b) Der Patient wird an der OP-Schleuse auf einen Transportwagen gelagert und von dort in den OP oder die Vorbereitung gefahren, um hier ein zweites Mal umgelagert zu werden.

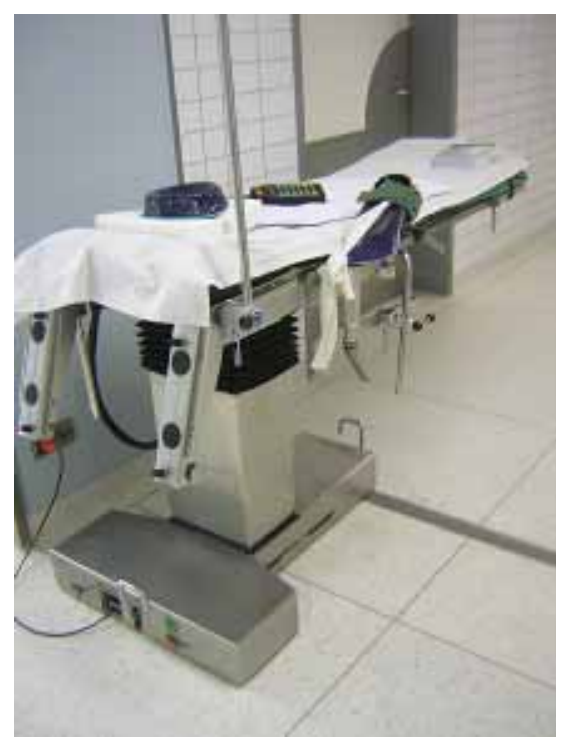

Abb.1 Operationstisch als eine Einheit mit Fahrgestell, Säule und Lagerungsfläche.
Bei der jetzigen OP-Tischgeneration ist Säule und OP-Tischauflage getrennt. Möglich ist dies durch technisch sehr ausgereifte konische Verbindungsmechanismen geworden (Abb.2). Diese müssen sich fest ineinander verbinden, damit Auflage und Säule eine Einheit werden. Die Verbindungen müssen auch noch die elektrischen Verbindungen zu den Motor getriebenen Gelenken tragen. Hier sind hohe Standards notwendig, um nicht zu Kurzschlüssen und anderen elektrischen Schäden auch unter schwierigen Bedingungen mit tropfenden Flüssigkeiten zu kommen.

Die OP-Tischauflage selbst wird durch eine Transportlafette bewegt.

Ein OP-Tischsystem besteht im Regelfall aus einer Säule, zwei Auflagen und zwei Transportlafetten. Die Auflage wird von einer Transportlafette zwischen OPSchleuse/Patientenübergabe zum OPTisch bewegt. Diese Transportlafetten können nun Fahrgestelle mit großen Rädern haben, welche den OP-Boden wesentlich weniger beanspruchen. Die OPLafette selbst ist mechanisch so ausgebildet, dass mit ihr das Aufsetzen und Einparken in die konische Verbindung zur OP-Tischsäule möglich ist.

Die Lafetten haben bei beweglichen Säulen auch die Aufgabe, diese vom Boden abzuheben (zum Transport und Reinigen).

\section{OP-Tisch-Säulen}

Wir kennen zwei Arten von OP-TischSäulen: nämlich die fest im Boden eingebaute (Abb.3) und die bewegliche (Abb.4).

\section{Feste Säule}

Die feste Säule ist im OP-Boden und zentral unter der Lüftungsdecke installiert. Mit der Auflage kann die Säule diagonal, 90 Grad oder 180 Grad gedreht werden. 


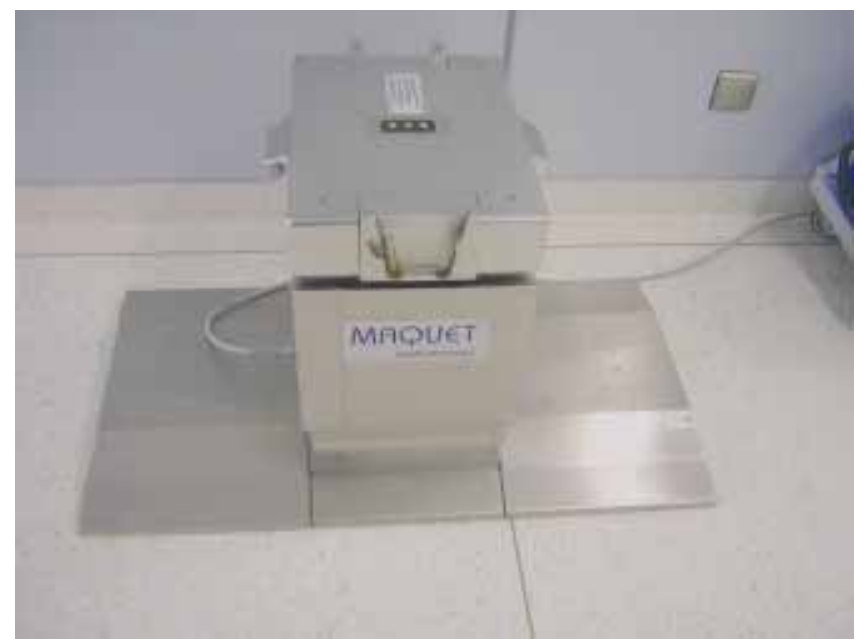

Abb. 2 Konischer Verbindungsmechanismus zwischen moderner OP-Säule und Lagerungsfläche.

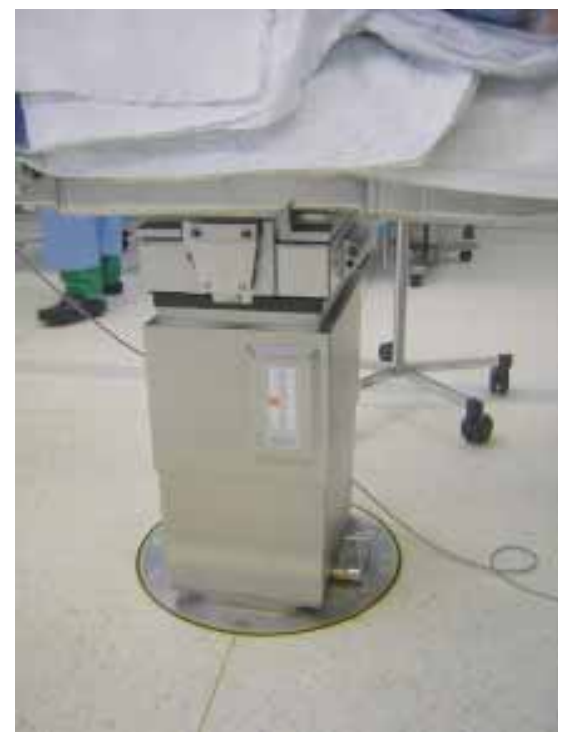

Abb. 3 „Feste“ Säule - im Boden eingebaut.

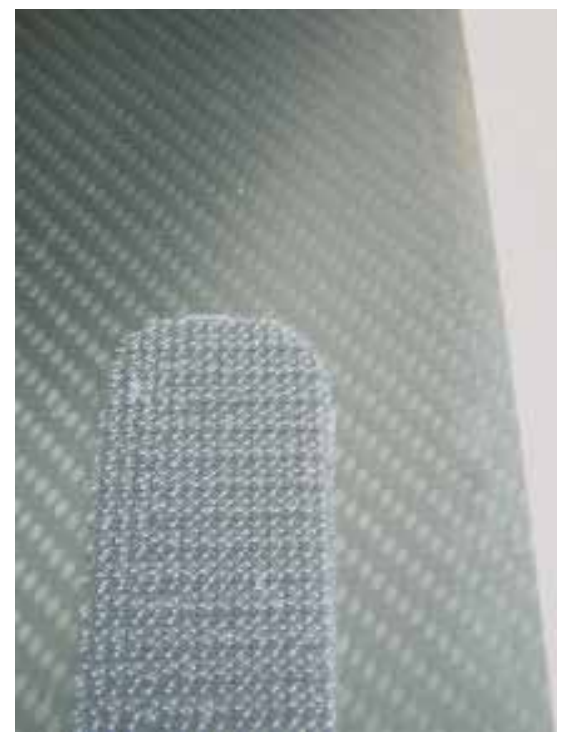

Abb. 4 Kohlefasertisch für die 3D-Bildwandlerbenutzung unablässig.

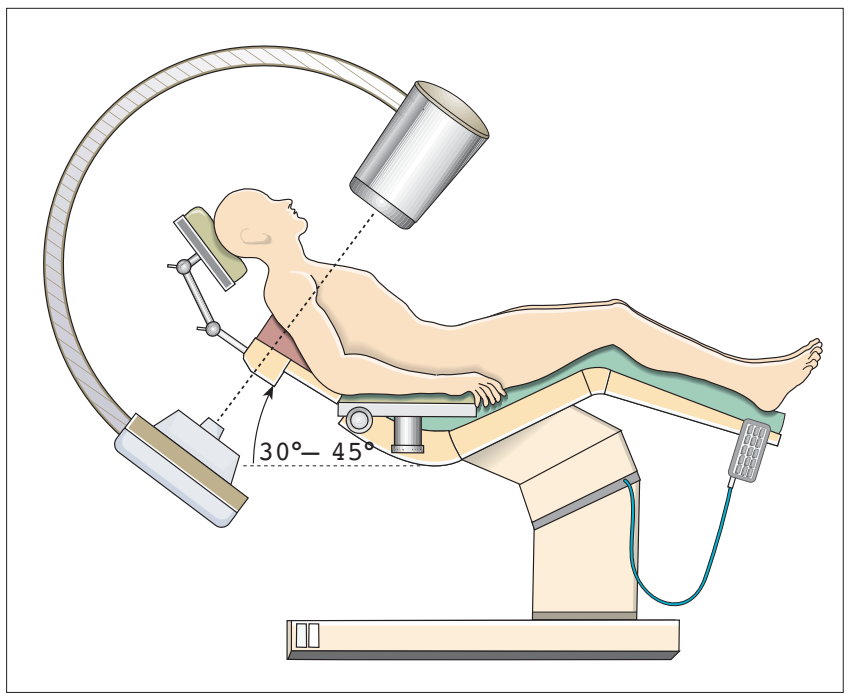

Abb. 5 BeachChair-Lagerung für die Operation an der Schulter.
Diese festen Säulen sind sehr tragfähig und erlauben damit weite Überhänge der Auflagen und sie sind z.B. notwendig bei weit überhängenden Tischen wie Kohlefasertischen für die 3D-Bildwandlerhandhabung (Abb.5).

Es könnte Folgendes passieren:

Eine OP-Abteilung hat sich wegen der größeren Flexibilität für bewegliche Säulen entschieden. Nun will man einen 3DBildwandler und die dazugehörige Kohlefaserliege anschaffen und stellt fest, dass die vorgesehenen Operationssäle nicht mit festen Säulen bestückt sind, wie es für die „durchsichtige“ C-Fasertische notwendig ist. Bei OP-Planungen für die $\mathrm{Zu}$ kunft ist dies zu berücksichtigen.

Feste Säulen sind hygienisch einwandfrei, und Boden und Säulen können gut gereinigt werden.

Eine asymmetrische Aufstellung ist nicht möglich.

Bei speziellen Operationen könnte dies von Vorteil sein, z. B. wenn auf der Operationsseite sehr viele Instrumententische benötigt werden. Manchmal wäre auch ein Verschieben nach kopf- oder fußwärts erwünscht (Operation am Hals und Kopf). Bei großen Überhängen und großen Patienten kann es passieren, dass diese Operationsregion nicht mehr ausreichend im Zentrum des Laminarflows der Lüftungsdecke liegt.

\section{Bewegliche Säulen}

Bewegliche Säulen sind in der Aufstellung flexibler. Sie können neben diagonaler Aufstellung auch seitwärts, kopfwärts und fußwärts bewegt werden. Am Kopf und Fuß gelegene Operationsfelder können besser unter die Lüftungsdecke zentriert werden. Auch hat der Anästhesist manchmal mit seinem immer größer werdenden Equipment mehr Platz.

Ein großer Nachteil ist oben beschrieben: Kohlefasertische mit großen Überhängen können nicht aufgesetzt werden. Unter den beweglichen Säulen muss täglich geputzt werden und zwar nicht nur der Fußboden, sondern auch die Unterseite der Säule. Dazu müssen diese OP-Säulen nach Eingriffen mit viel Flüssigkeitsfluss (Arthroskopie oder starkes Bluten) oder auf jeden Fall am Ende eines Operationstages vom Reinigungspersonal entsprechend bedient werden können. 
Folgende OP-Tisch-Konfigurationen sind heute in Anwendung:

- OP-Tisch „aus einem Stück“ mit Fahrgestell, Säule und Patientenlagerungsfläche

- Bewegliche Säulen mit gesonderten Lagerungsflächen

- Feste Säulen mit gesonderten Lagerungsflächen

Für jede der genannten Konfigurationen gelten gesonderte Bedingungen für die Logistik.

\section{Fernbedienung}

Zur Bedienung von festen und beweglichen Säulen werden heute gern drahtlose Fernbedienungen gewählt. Diese müssen genau beherrscht werden. Häufig ist es so, dass verschiedene OP-Tisch-Säulen verschiedene Fernbedienungen haben, so dass eine Verwechselung vermieden werden muss. Es muss auch immer auf einen Ladezustand der Akkus der Fernbedienung geachtet werden.

\section{OP-Tisch-Lagerungsflächen}

Die Auflageflächen von OP-Tischen sind heute technisch sehr ausgereift und diversifiziert. Es gibt sie in großer Vielfalt und Variabilität, auch für die verschiedenen Fachrichtungen wie Unfallchirurgie, Orthopädie, Gynäkologie, Urologie usw.

Für die Unfallchirurgie und Orthopädie kommt im Regelfall eine Lagerungsfläche zur Anwendung, welche nach distal in der Mitte längs geteilt ist und nach proximal mehrere Flächenelemente nacheinander aufreiht. So können verschiedene gleichsinnige und gegenläufige Knickungen erreicht werden (Abb. 5). Z. B. mittiger oder asymmetrischer Knick für die Seitenlage bei Wirbelsäulenoperationen oder z.B. Beach-Chair (Liegestuhl)-Lagerung für die Schulterchirurgie. Es sind ebenso verschiedene Abknickungen im Hüft- und Kniegelenksbereich (z.B. Arthroskopie oder Nagelung am hängenden Bein) möglich.

Für die spezielle Schulterchirurgie gibt es spezielle Tischplatten-Konfigurationen. Bei etwas asymmetrischer Lagerung und Fassen des Kopfes in einer Stütze kann dann das Tischteil unter der zu operierenden Schulter entfernt werden (Abb.5). So kann weit nach dorsal abgedeckt werden und die BV-Kontrolle ist einfacher (s.u.).

Weitere typische Lagerungen werden bei der Lagerung erklärt.

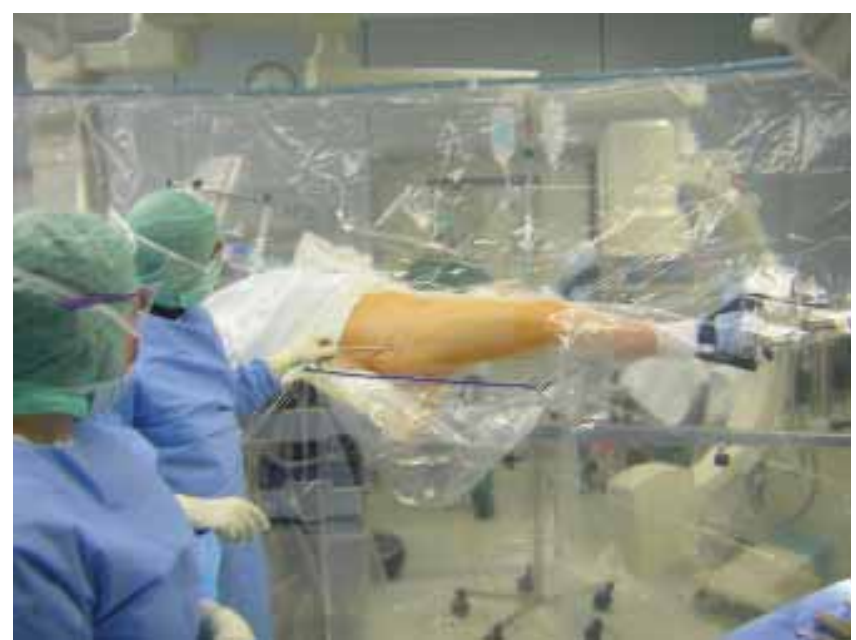

Abb. 6 Extensionstisch mit Abdeckung für Operationen am proximalen Oberschenkel.

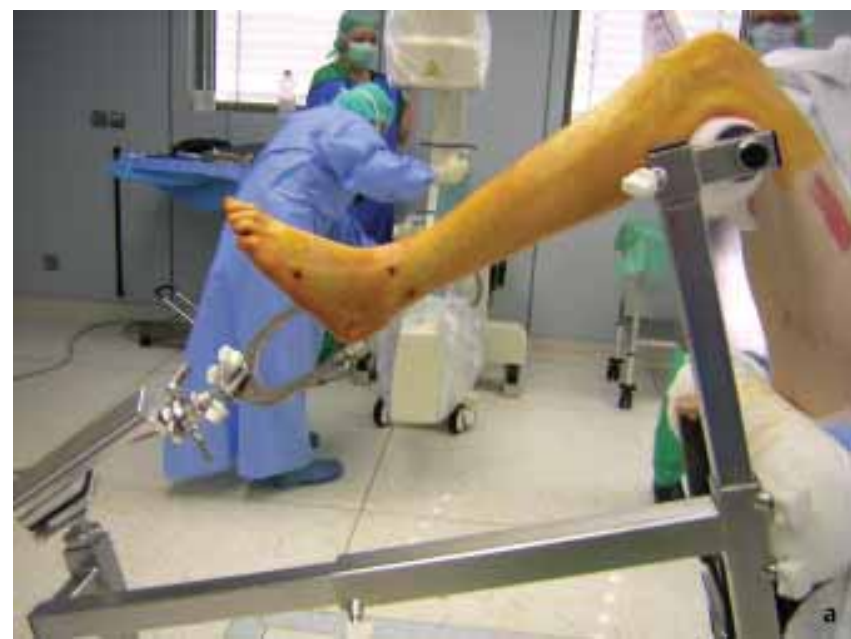

Abb.7 Extensionstisch für die Unterschenkelmarknagelung.

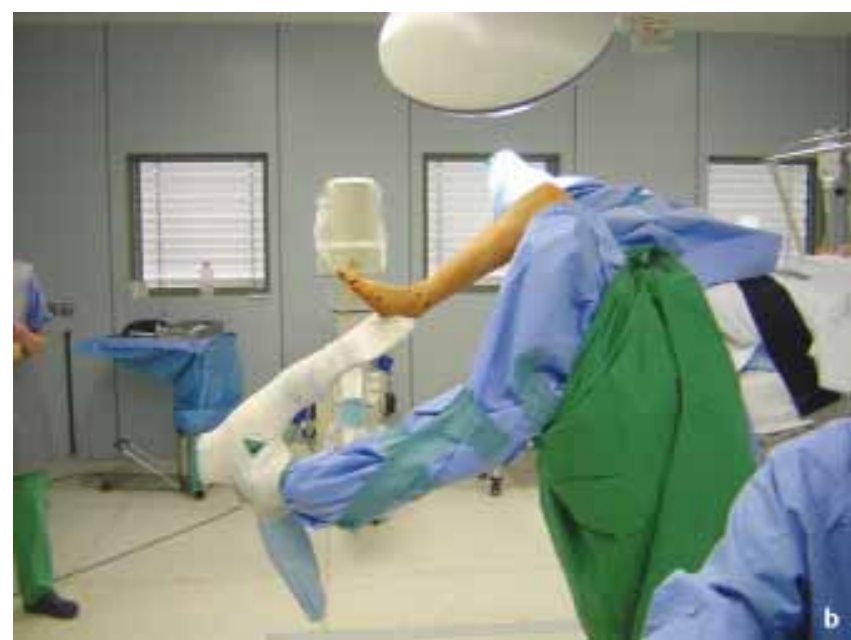

Die Lagerflächen erlauben es, verschiedene Anbauten entlang der längs verlaufenden Schienen oder an den Enden (vor allem Kopfende) anzubringen. Genannt werden sollen: Extensionstisch (gestreckt für den Oberschenkel (Abb.6) oder im Kniegelenk gebeugt für den Unterschen- kel) (Abb. 7), Kopfschalen, Kopfhalterungen (z.B. Mayfield-Klemmen usw.) (Abb.8). Die Handhabungen all dieser Zusätze müssen geübt und beherrscht werden. Für seltene Anwendungen haben sich schriftliche und bildliche Anleitungen bewährt. 


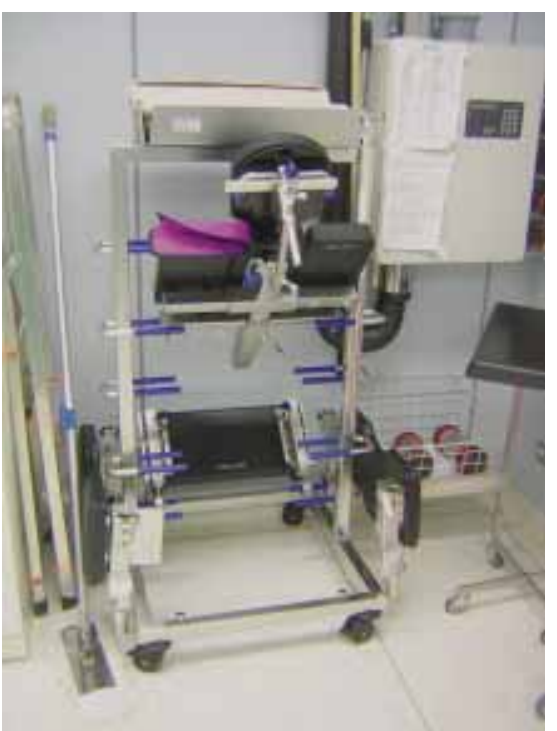

Abb. 8 Zusatzgeräte mit Kopfhalterung usw.

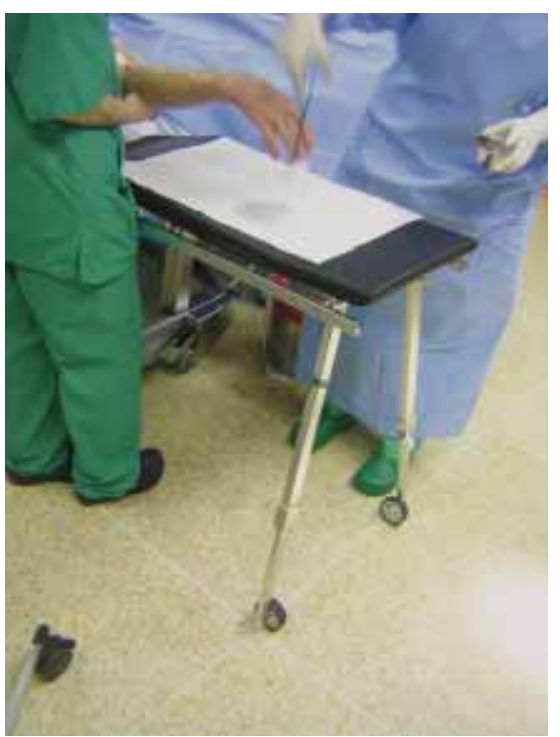

Abb.9 An den OP-Tisch anhängbare Handtische.

Die OP-Tische werden noch ergänzt durch arretierbare Handtische (Abb.9), Oberarmauflagen (z.B. für Operation am Oberarm in Bauchlage, s.u. und Abb.10).

Eine neue Art der Lagerfläche ist die Kohlefaserauflage (Abb.4). Diese ist für die Röntgenstrahlen des Bildwandlers vollkommen durchlässig. Es entstehen im BV-Bild keine Schatten und seitlich stören keine Metallschienen. Diese Auflage ist vor allem notwendig für seltene Anwendungen des CTs im OP, aber vor allem für die sich rasch ausbreitende 3D-Bildwandlertechnik (Siemens Iso 3C und Ziehm Vario 3D).

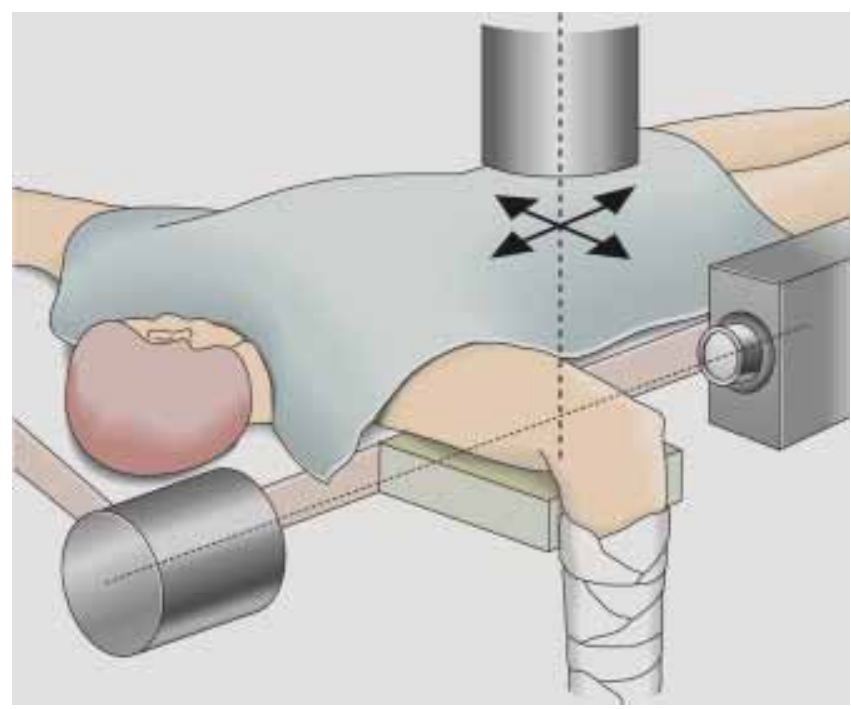

Abb.10 Lagerung für die retrograde Marknagelung oder dorsale Plattenosteosynthese am Oberarm.

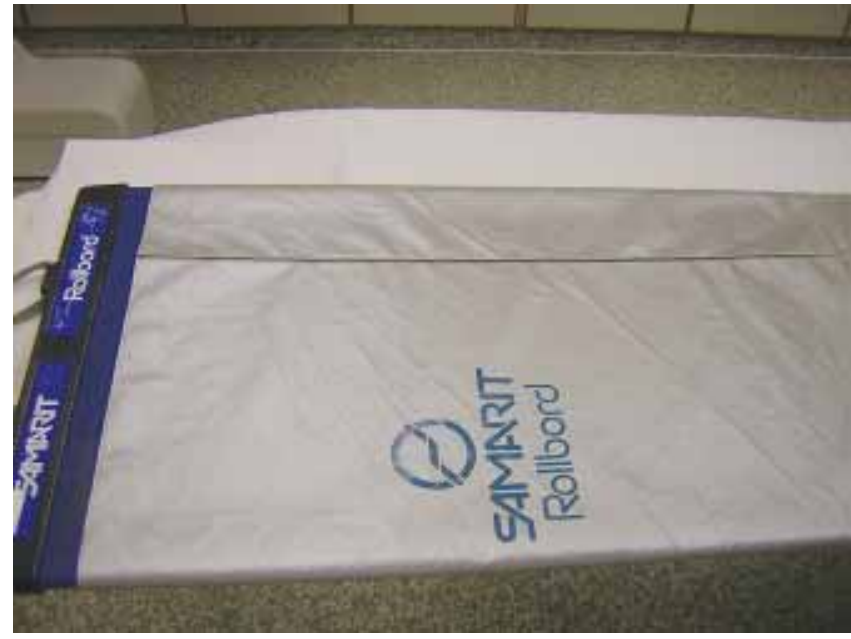

Abb.11 Rollbrett zum Umlagern von Patienten.

Diese sehr gut für Röntgenstrahlen durchsichtigen Lagerflächen haben sich aber auch bei Wirbelsäulen und Beckeneingriffen ohne 3D-Bildwandlertechnik bewährt. Durch die schattenfreie und störungsfreie Abbildung werden die intraoperativen BV-Bilder wesentlich besser. So ist z.B. die Ortung der Pedikel im Wirbelsäulenbereich und die Ortung der anatomischen Landmarken bei Beckenoperationen, z.B. Verschraubung der Iliosakralfuge (minimalinvasiv), wesentlich leichter, weil die Röntgen-BV-Bilder klarer und ungestörter sind.

Bei der Anschaffung eines 3D-Bildwandlers ist zu beachten, dass neben dem Anschaffungspreis für den 3D-BV auch noch der Anschaffungspreis von mehreren $10 €$ für die Kohlefaserliege im Budget mit berücksichtigt werden muss.

Die OP-Tisch-Lagerflächen werden dann noch durch die sog. Tischauflagen gepolstert. Hier haben sich Gelmatratzen
(Tempura-Matratzen) sehr bewährt. Hiermit werden weiche nachgiebige Auflagen mit doch genügend stabiler Härte kombiniert.

Lagerungsflächen:

- Sie sind heute hoch komplex und variantenreich. Jeder Operationsmethode kann eine spezielle Lagerungsfläche zugeordnet werden.

- Die OP-Koordination muss für elektive Eingriffe des OP-Programms sowie für Notfalleingriffe die entsprechende organisatorischen Voraussetzungen schaffen.

- Neben den sehr variablen knickbaren Lagerungsflächen sind für besondere Anwendungen (3D-BV) nicht knickbare durchgehende Kohlefaserauflagen notwendig.

\section{Patientenvorbereitung}

An dieser Stelle soll von der Schnittstelle zwischen OP-Schleuse und OP-Tisch gesprochen werden. 


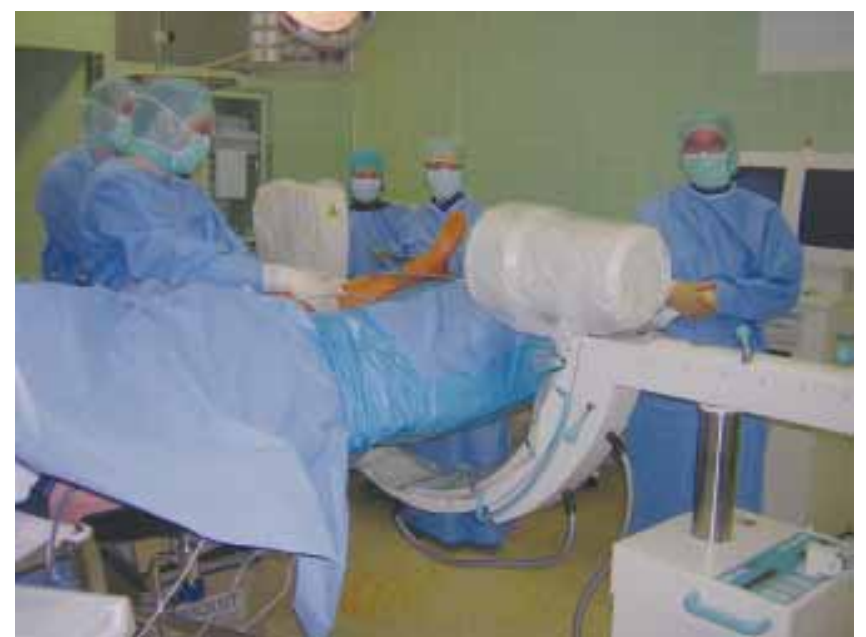

Folgendes ist zu beachten:

Vorsichtiges Umlagern von Hand mit entsprechenden Unterlagen (z.B. Save-Tex oder Rollbretter) (Abb.11) oder mit den mechanischen Umlagerungshilfen. Durch die Möglichkeiten des Save-Tex und der Rollmatratze werden die mechanischen Umlagerungshilfen nicht für alle Umlagerungen verwendet, so dass man eine gemischte Anwendung vorfindet. Dies wird auch von Abteilung zu Abteilung und nach den Vorlieben des OP-Personals variieren.

Der Röntgenschutz muss beachtet werden.

- Ein vollständiger Röntgenschutz muss rundherum gegeben sein, so dass die entsprechende Matte unter und auf dem Patienten liegen sollte.

Bei den entsprechenden Eingriffen ist zwischen OP-Pflege, Lagerungsteam und den Operateuren genau abzusprechen, wie der Bildwandlerdurchlauf ist. Für schwierige und besondere Eingriffe hat es sich bewährt, dass der Bildwandlerdurchlauf in Übungen trainiert wird, und dass nach der Lagerung des Patienten vor dem sterilen Abdecken von Bildwandler und Patient dies einmal vom Operateur geprüft wird.

- Bei längeren Eingriffen sind Wärmematten und Wärmedecken zu verwenden.

Von den weiteren Patientenvorbereitungen wie arterielle und venöse Zugänge, Dauerkatheter, Magensonde usw. soll hier nicht näher eingegangen werden.

Die Patientenvorbereitung mit Rasieren und Waschen der Extremitäten bei frischen Verletzungen muss in jeder Abtei-
Abb.12 Steril eingepacktes Fußende, um den Durchlauf des Bildwandlers (steril) zu erleichtern.

lung für sich festgelegt werden, wobei es hier durchaus erhebliche organisatorische Unterschiede geben kann. Wichtig ist, dass die Prozedur für eine Abteilung einheitlich, transparent und reproduzierbar festgelegt und durchgeführt wird.

Die Patientenvorbereitung ist eine der verantwortungsvollsten Aufgaben des OP-Pflegepersonals, um für die Operation bestmöglichste und sicherste Voraussetzungen zu gewährleisten.

\begin{tabular}{|c|c|}
\hline \multicolumn{2}{|l|}{ Lagerung } \\
\hline - normale Rückenlagerung & $\begin{array}{l}\text { Osteosynthesen obere und untere Extremitäten } \\
\text { Abdomen und Thoraxeingriffe } \\
\text { Beckenoperationen von ventral (ilioinguinaler Zugang, Sym- } \\
\text { physe, Fixateur externe) mit aufgestelltem Unterschenkel für } \\
\text { Tibiamarknagelung }\end{array}$ \\
\hline $\begin{array}{l}\text { - Rückenlagerung } \\
\text { „mit ausgelagertem Ober- } \\
\text { schenkel“ }\end{array}$ & Marknagelung Oberschenkel \\
\hline $\begin{array}{l}\text { „mit Kopfschale oder } \\
\text { Kopfhalterung“" }\end{array}$ & Operation an der HWS von ventral \\
\hline \multirow{3}{*}{$\begin{array}{l}\text { - Extensionstisch } \\
\text { Rückenlage }\end{array}$} & Marknagelung Oberschenkelschaft \\
\hline & Osteosynthese proximaler Oberschenkel (Abb. 6) \\
\hline & Marknagelung Unterschenkel bei gebeugtem Knie (Abb. 7) \\
\hline $\begin{array}{l}\text { - Beach-Chair-Lagerung } \\
\text { (Abb. 5) }\end{array}$ & $\begin{array}{l}\text { Schultereingriffe } \\
\text { anterograde Marknagelung des Oberarms }\end{array}$ \\
\hline - Seitenlage & $\begin{array}{l}\text { Azetabulum mit Kocher-Langenbeck-Zugang und erweiterte } \\
\text { Zugänge } \\
\text { Wirbelsäuleneingriffe BWS und LWS } \\
\text { thorakoskopisch, lumbal, minimalinvasiv oder offen } \\
\text { Fersenbeinfrakturen } \\
\text { Schultereingriffe (Arthroskopie) }\end{array}$ \\
\hline - Bauchlage & $\begin{array}{l}\text { Osteosynthesen der Wirbelsäule von dorsal am dorsalen } \\
\text { Beckenring } \\
\text { Weichteileingriffe von dorsal } \\
\text { Baker-Zyste } \\
\text { Osteosynthesen am Tibiakopf von dorsal } \\
\text { retrograde Marknagelung des Oberarms } \\
\text { dorsale Plattenosteosynthesen Oberarmschaft } \\
\text { dorsale Osteosynthesen/Kondylenfrakturen des Oberarms }\end{array}$ \\
\hline
\end{tabular}

\section{Lagerungstechniken}

In einem 10-jährigen Jubiläumsheft dieses OP-Journals vor genau 10 Jahren ist ein Großteil der Lagerungstechniken von Schulter bis Fuß beschrieben [1] Viele dieser beschriebenen Techniken haben noch heute Bestand, so dass es sich lohnt, dieses OP-Journal aus dem Jahr 1995 noch einmal zu lesen.

Die Operationslagerung [2] ist bei vielen Eingriffen ein entscheidendes Kriterium für einen harmonischen Ablauf der Operation, und vielfach ist die richtige Lagerung auch Voraussetzung für den korrekten Zugang und Operationsmöglichkeit.

Eine richtige Lagerung erleichtert die Operation wesentlich, eine falsche kann sie sehr erschweren und damit risikoreicher machen oder gar verunmöglichen.

Die verschiedenen Lagerungsformen mit typischen Operationsindikationen für Unfallchirurgie und Orthopädie sollen im Folgenden tabellarisch aufgelistet und wesentliche, besonders häufige Lagerungen abgebildet werden. 
Die Lagerung muss auch mögliche intraoperative Komplikationen und/oder Erweiterungen der Operation berücksichtigen.

\section{Tipps und Tricks}

Aus der Erfahrung in der Klinik des Autors sollen ärztlichen Kollegen und Teamkollegen des OP-Personals einige pragmatische Tipps gegeben werden, welche sich in Tübingen bewährt haben.

- Steriles Einpacken des freien überstehenden Endes des OP-Tisches, um den freien sterilen Durchlauf des Bildwandlers $\mathrm{zu}$ erlauben. Aus unserer Sicht erleichtert dies die intraoperative BV-Handhabung wesentlich (Abb.13).

- Für die Abdeckung auf dem Extensionstisch für die operative Versorgung von proximalen Femurfrakturen hat sich die Folienwand mit zentraler Klebefläche sehr bewährt (Abb.6).

- Bei komplexen Frakturen an einer unteren Extremität hat es sich bewährt, beide Beine steril abzudecken (Abb.13). Dadurch hat man für die zu operierende Extremität einen sehr einfachen natürlichen Vergleich, um Länge, Rotation und Achse einzustellen. Gleichzeitig kann durch Manipulation des gesunden Beines die Bildwandlerdarstellung im seitlichen Strahlengang durch Anheben dieses Beines wesentlich vereinfacht werden. Sehr bewährt hat sich dies bei den eingeschobenen Plattenosteosynthesen (LISS) aber auch bei der Marknagelung mit einfacher Rückenlagerung.

- Der distale Femurnagel kann mit hälftig abgenommener Fußstütze operiert werden. Das Kniegelenk fällt automatisch in $90^{\circ}$. Kein herabgelassenes Fußende stört den Bildwandlerdurchgang, insbesondere im so wichtigen Kniegelenksbereich.

Wenn zum Abschluss der Operation das Bein gestreckt werden soll, kann dies auf einen fahrbaren Zusatztisch, welcher auf gleicher Höhe wie der Operationstisch eingestellt ist, gelegt werden.

- Für die Humerusnagelung von distal in Bauchlage verwenden wir die im Haus schon vorhandene Kohlefaserliege. Der

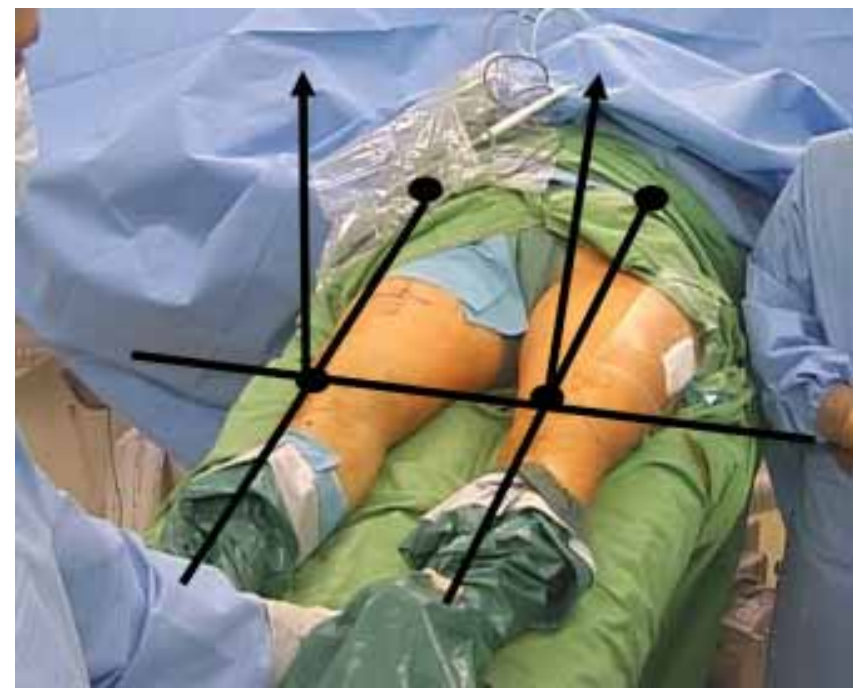

Abb.13 Simultan abgedeckte Beine für die Operation von komplexen Ober- und/oder Unterschenkelfrakturen.

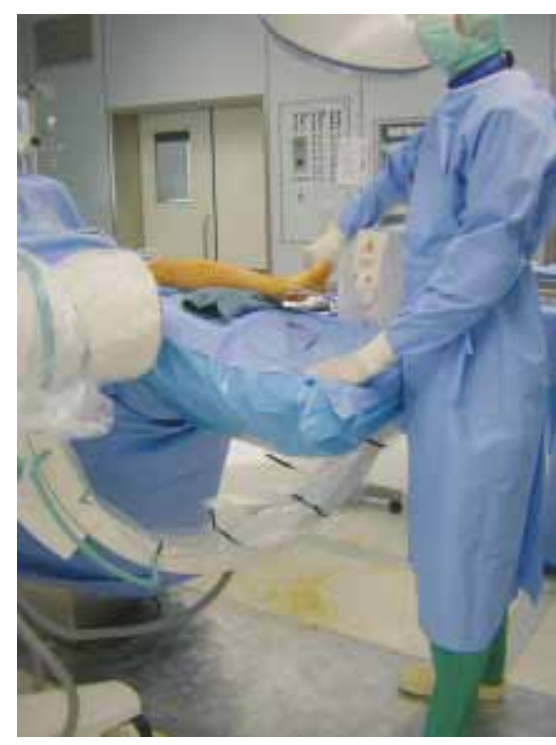

Abb.14 Seitenlagerung zur Operation von Fersenbeinfrakturen.

Arm wird auf ein ebenfalls Röntgenstrahlen „durchsichtiges“ Kohlefaserbrett gelegt. Dadurch stört kein Tischelement die proximale Verriegelung.

- Für Operationen im Beckenbereich und an der oberen Brustwirbelsäule nehmen wir ebenfalls die Kohlefaserliege, auch wenn wir keine 3D-Bildwandlerdarstellung vornehmen. Die intraoperative BV-Kontrolle ist wesentlich besser und erleichtert das Erkennen von Landmarken für die Operation.

Kurze Zusammenfassung von Tipps und Tricks:

- Einpacken des OP-Tischendes

- Simultanes Abdecken beider Beine

- $90^{\circ}$ Beugung bei fallendem Unterschenkel für den DFN

- Häufiges Verwenden des Kohlefasertisches bei Operationen am Stammskelett

\section{Literatur}

${ }^{1}$ Jockheck M, Höntzsch D. Lagerungen für Operationen. OP Journal (Sonderausgabe): 1995; $32-58$

2 Rüedi TP, Murphy WM. AO Principles of Fracture Management. Thieme Verlag New York Stuttgart 2000

Prof. Dr. med. Dankward Höntzsch Leitender Arzt

B6-Unfallklinik

Schnarrenbergstraße 95

D-72076 Tübingen 